\title{
Search for the Charmonium Dissociation Temperature with Variational Analysis in Lattice QCD
}

\author{
Hiroshi Ohno*, Takashi Umeda, and Kazuyuki Kanaya (WHOT-QCD Collaboration) \\ Graduate School of Pure and Applied Sciences, University of Tsukuba, Tsukuba, Ibaraki \\ 305-8571, Japan \\ E-mail: ohno@het.ph.tsukuba.ac.jp
}

\begin{abstract}
Charmonium dissociation temperatures are studied in a quenched anisotropic lattice QCD with standard plaquette gauge action and $O(a)$ improved Wilson fermion action. Simulations are carried out at temperatures in the range $0.88 T_{c}$ to $2.3 T_{c}$. From the meson correlators, we first subtract the contribution of constant mode, which was reported to mislead the analysis of, in particular, $\mathrm{P}$ wave signals. We then calculate effective masses and Bethe-Salpeter wave functions for ground (1S, 1P) and excited states (2S, 2P) using the multi-state variational analysis. To distinguish between bound states and scattering states, we apply two methods: First, we compare effective masses for charmonium correlation functions with finite spatial momenta under different spatial boundary conditions. Since the scattering state energies are sensitive to the boundary conditions, we expect finite volume effects when the charmonium dissociates. Second, we study the Bethe-Salpeter wave function, which should become broad when the charmonium bound state turns into a scattering state. With both methods, we have fond no clear evidences of dissociation for the ground and exited charmonium states up to $2.3 T_{c}$ so far.
\end{abstract}

The XXVI International Symposium on Lattice Field Theory

July 14 - 19, 2008

Williamsburg, Virginia, USA

\footnotetext{
* Speaker.
} 


\section{Introduction}

Studying heavy quarkonia properties above $T_{c}$ is important to understand how quark-gluon plasma (QGP) is formed in heavy ion collisions. In particular, dissociation of charmonia in deconfined phase is an interesting subject because $J / \psi$ suppression [1] is one of the most important signals of QGP. Recently, the sequential $J / \psi$ suppression scenario [2], in which dissociation of heavier charmonia $\left(\chi_{c}, \psi^{\prime}\right)$ play important roles for the suppression of total $J / \psi$ yield, has been proposed to understand the experimental results in heavy ion collisions at SPS and RHIC.

Lattice QCD studies of charmanium spectral function using the Maximum Entropy Method (MEM) suggested that $S$ wave charmonia, such as $\eta_{c}$ and $J / \psi$, survive up to $1.5 T_{c}$ [3, 4, 5, 6, , , , while $\mathrm{P}$ wave charmonia, such as $\chi_{c}$ 's, dissociate just above $T_{c}$ [6, 7, 8].

Although these results look supporting the sequential $J / \psi$ suppression scenario, there are still some problems. First, the meson correlators receive sizable contribution of the constant mode above $T_{c}$, especially in $\mathrm{P}$ wave correlators 9 . From an analysis in which the constant mode contribution was properly taken into account, the thermal effects in charmonium correlators including those of $\mathrm{P}$ waves turned out to be quite small up to $1.4 T_{c}$. This is in contrast to the conclusion of previous MEM studies on $\mathrm{P}$ wave charmonia. Furthermore, choice of the default model for the spectral function is known to affect the MEM results rather sensitively. A crosscheck without Bayesian-type analyses is needed.

Let us first discuss what we expect on the lattice when a charmonium dissociates. It should be noted that, on a lattice with finite extent, spectral functions consist of discrete spectra only, also at $T>T_{c}$. (These discrete spectra appear as broad peaks in approximate calculations such as MEM.) Below $T_{c}$, we expect to have discrete peaks corresponding to ground and exited bound states. When a charmonium bound state fully dissolves above $T_{c}$, the peak corresponding to the bound state will vanish and other peaks corresponding $c-\bar{c}$ scattering states may appear near the vanished peak. Furthermore, we expect that the wave function for the peak will have a localized shape when the peak corresponds to a bound state, while it will have a broad shape extending to large distances when the peak corresponds to a scattering state.

In this paper, we report on our study of charmonium dissociation. In principle, when a high precision data of the correlation function is available up to large distances, the constant mode can be identified by conventional analyses too. In practice, however, with current accuracy and the range of sensible data, we think that it is safer to explicitly subtract out the contribution of the constant mode from the correlators. We apply the midpoint subtraction method developed in [9]. Avoiding Bayesian-type analyses, we then adopt the multi-state variational method to extract ground and exited state masses both in $\mathrm{S}$ and $\mathrm{P}$ waves [10]. At the same time, we calculate the Bethe-Salpeter wave functions of these states from the spatial correlation function between $c-\bar{c}$ quarks [3].

To distinguish bound states of $c-\bar{c}$ quarks from their scattering states for the extracted states, we study the spatial boundary condition dependence of the energy spectrum at finite volume [1]: The energy of scattering states depends on its relative momentum which is quantized according to the spatial size and boundary conditions. On the other hand the spectrum of the bound states does not change against such exchange of boundary conditions. The Bethe-Salpeter wave functions for a bound state will be compact, while those for scattering state will extend and will change its shape depending on the spatial lattice size. Combining these tests, we may examine if the charmonium is 
Table 1: The parameter $A_{i}$ of the smearing functions.

\begin{tabular}{|c||c|c|c|c|c|c|}
\hline$i$ & 1 & 2 & 3 & 4 & 5 & 6 \\
\hline$A_{i}$ & 0.02 & 0.05 & 0.10 & 0.15 & 0.20 & 0.25 \\
\hline
\end{tabular}

dissociated.

\section{Multi-state variational analysis}

The charmonia correlation matrix in Euclidian space-time is defined by

$$
C_{i j}(t) \equiv \sum_{\vec{x}}\left\langle O_{i}^{\Gamma}(\vec{x}, t) O_{j}^{\Gamma}(\overrightarrow{0}, 0)^{\dagger}\right\rangle
$$

with $\Gamma=\gamma_{5}, \gamma_{i}, \mathbf{1}(i=1,2,3)$ for pseudo-scalar (Ps), vector (Ve) and scalar (Sc) channels, respectively. Here $\left\{O_{i}^{\Gamma} \mid i=1,2, \cdots N_{\text {state }}\right\}$ are smeared meson operators defined by

$$
O_{i}^{\Gamma}(\vec{x}, t) \equiv \sum_{\vec{y}, \vec{z}} \omega_{i}(\vec{y}) \omega_{i}(\vec{z}) \bar{q}(\vec{x}+\vec{y}, t) \Gamma q(\vec{x}+\vec{z}, t),
$$

with Gaussian smearing functions $\omega_{i}(\vec{x}) \equiv \exp \left(-A_{i}|\vec{x}|^{2}\right)$. Table 1 shows our choice of the smearing parameters $A_{i}$.

According to [9], constant mode effects are large in the deconfined phase, because the constant mode is due to wraparound contributions of single quark propagators, which are suppressed in the confined phase. To separate out the constant mode contribution from meson correlators, we study midpoint subtracted correlators $\bar{C}(t)$ defined by

$$
\begin{aligned}
\bar{C}(t) & \equiv C(t)-C\left(N_{t} / 2\right) \\
& =\left(c_{0}+\sum_{k=1}^{\infty} c_{k} \cosh \left[m_{k}\left(t-\frac{N_{t}}{2}\right)\right]\right)-\sum_{k=0}^{\infty} c_{k} \\
& =2 \sum_{k=1}^{\infty} c_{k} \sinh ^{2}\left[\frac{m_{k}}{2}\left(t-\frac{N_{t}}{2}\right)\right] .
\end{aligned}
$$

Here $C(t)$ is the matrix of $C_{i j}(t)$ defined in 2.1 ,,$c_{0}$ is a contribution of the constant mode and $N_{t}$ is the temporal lattice size.

We extract ground and excited states in $\bar{C}$ by solving the eigenvalue problem,

$$
\bar{C}(t) \vec{v}_{k}=\lambda_{k}\left(t ; t_{0}\right) \bar{C}\left(t_{0}\right) \vec{v}_{k} \quad\left(k=1,2, \cdots, N_{\text {state }}\right) .
$$

Effective masses of the diagonalized states are given by

$$
\lambda_{k}\left(t ; t_{0}\right)=\frac{\sinh ^{2}\left[\frac{M_{k}^{\text {eff }}}{2}\left(t-\frac{N_{t}}{2}\right)\right]}{\sinh ^{2}\left[\frac{M_{k}^{\text {eff }}}{2}\left(t_{0}-\frac{N_{t}}{2}\right)\right]} .
$$


Table 2: The number of gauge configurations.

\begin{tabular}{|c||c|c|c|}
\hline & $N_{s}=16$ & $N_{s}=20$ & $N_{s}=32$ \\
\hline \hline local operator & 800 & 800 & 200 \\
\hline derivative operator & 300 & 300 & 200 \\
\hline
\end{tabular}

Next, we define wave functions in terms of the Bethe-Salpeter (BS) amplitude:

$$
\begin{aligned}
B S_{i}(\vec{r}, t) & \equiv \sum_{\vec{x}}\left\langle\bar{q}(\vec{x}, t) \Gamma_{\mathrm{snk}} q(\vec{x}+\vec{r}, t) O_{i}^{\Gamma_{\mathrm{scc}}}(\overrightarrow{0}, 0)^{\dagger}\right\rangle \\
& =\sum_{k=0}^{\infty}\left(\psi_{k}(\vec{r})\right)_{i} \cosh \left[m_{k}\left(t-\frac{N_{t}}{2}\right)\right] .
\end{aligned}
$$

Here $\left(\psi_{k}(\vec{r})\right)_{i}$ is the wave function for the $k$-th state created by the smeared source operator $O_{i}^{\Gamma_{\text {src }}}$. To study $S$ waves, we adopt $\Gamma_{\text {src }}=\Gamma_{\text {snk }}=\gamma_{5}$ and $\gamma_{i}$ for Ps and Ve charmonia, respectively. For Sc charmonium in P wave, we use a derivative source $\Gamma_{\mathrm{src}}=\gamma_{i}\left(\overleftarrow{\partial_{i}}-\overrightarrow{\partial_{i}}\right)$ to enhance the signal, with $\Gamma_{\text {snk }}=\gamma_{i}$.

To seprate out the constant mode, we define midpoint subtracted BS amplitudes by

$$
\overline{B S}(\vec{r}, t) \equiv B S(\vec{r}, t)-B S\left(\vec{r}, N_{t} / 2\right) .
$$

Finally, the diagonalized wave functions are given by

$$
\Psi_{k}\left(\vec{r}, \vec{r}_{0}\right) \equiv \frac{\sum_{i} \overline{B S}_{i}(\vec{r}, t) V_{i k}}{\sum_{i} \overline{B S}_{i}\left(\vec{r}_{0}, t\right) V_{i k}}
$$

where $V_{i k} \equiv\left(v_{k}\right)_{i}$ is the $i$-th component of the eigen vector $\vec{v}_{k}$, and $\vec{r}_{0}$ is the normalization point.

\section{Numerical results}

\subsection{Lattice setup}

We study quenched QCD with the anisotropy $\xi \equiv a_{s} / a_{t}=4$. The gauge action is the standard plaquette action with $\beta=6.10$ and a bare anisotropy parameter $\gamma_{G}=3.2108$. For quarks, we adopt $O(a)$ improved Wilson fermion action with tadpole-improved tree-level clover coefficients. Our actions and their parameters are the same as those in [12] except for the choice of the Wilson parameter, $r=1$. The spatial lattice spacing is $a_{s}^{-1}=2.030(13) \mathrm{GeV}$. Simulations are performed on $N_{s}^{3} * N_{t}$ lattice, with $N_{s}=16,20$ and 32 and $N_{t}=32,26,20,16$ and 12, which correspond to $T=\left(N_{t} a_{t}\right)^{-1}=0.88 T_{c}, 1.1 T_{c}, 1.4 T_{c}, 1.8 T_{c}$ and $2.3 T_{c}$ respectively. (Critical temperature $T_{c}$ corresponds to $N_{t} \simeq 28$.) The number of gauge configurations is shown in Table 2 . We choose the Coulomb gauge fixing to calculate the wave functions.

\subsection{Effective masses}

In Figure 1 we compare the ground state's and the first excited state's effective masses of Ve and Sc charmonia as functions of $T$ between different boundary conditions, i.e. periodic (PBC), 

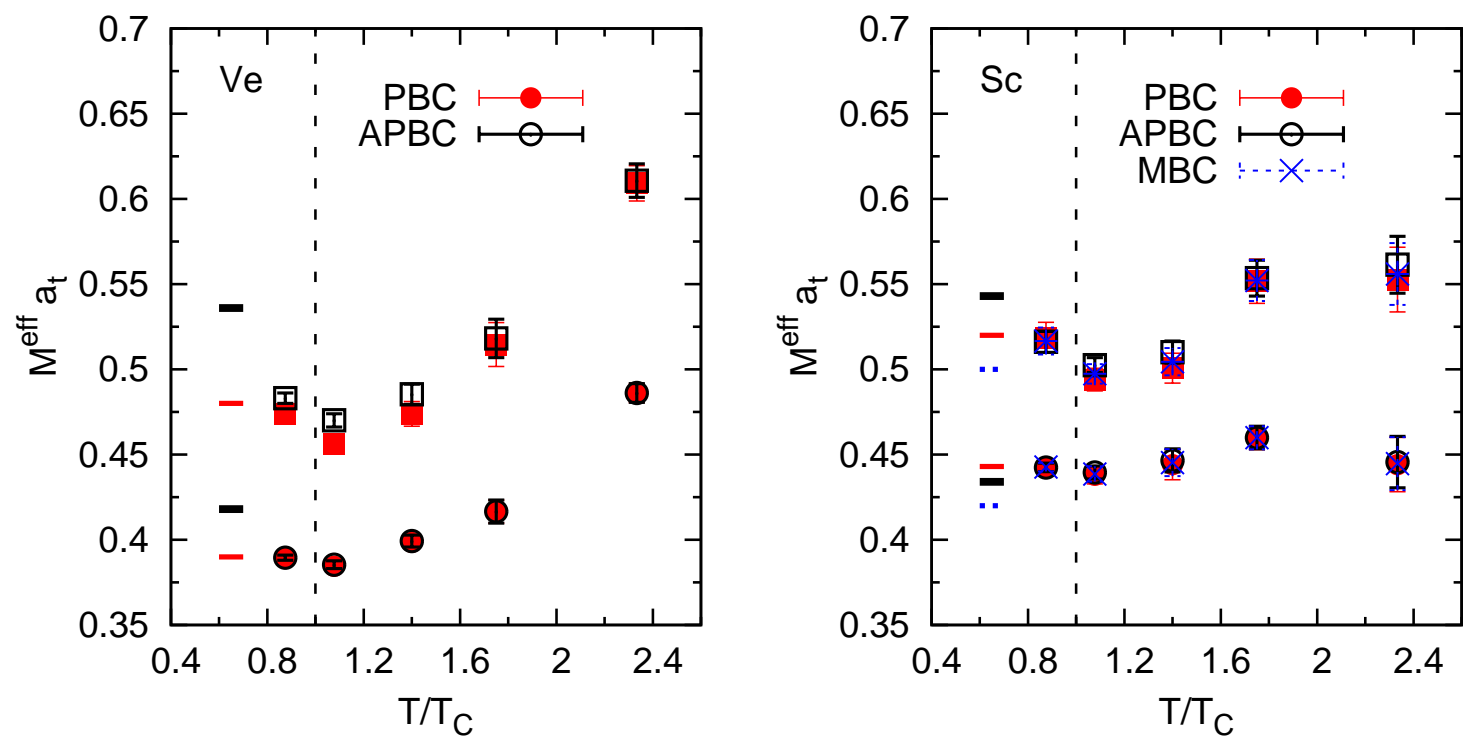

Figure 1: The effective masses for Ve (left) and Sc (right) charmonia. Lower and upper symbols correspond to the ground and first excited states, respectively. Red, black and blue symbols indicate the effective masses with PBC, APBC and MBC, respectively (see text for the definition of MBC). Short bars shown near the left end of each figure indicate the boundary condition dependence of energy spectra for the case of free quarks.

anti-periodic (APBC) and a mixed (MBC) boundary conditions. Here MBC is defined by APBC in the $x$-direction combined with PBC in $y$ and $z$-directions. At zero temperature, the ground state and the first excited state of Ve channel correspond to $J / \psi$ and $\psi^{\prime}$ respectively, and the ground state of Sc channel corresponds to $\chi_{c 0}$. The effective masses are calculated by the multi-state variational analysis with $4 \times 4$ correlation matrix on the $20^{3} \times N_{t}$ lattice. Here we choose smeared operators with the parameter $A_{3}, A_{4}, A_{5}$ and $A_{6}$ of Table 1 to obtain the largest overlap with the ground state and the first excited state.

To see a typical magnitude of the boundary-condition dependence in the effective masses, we have studied the energy spectra for the case of free quark in a box of $(2 \mathrm{fm})^{3}$, assuming that the charm quark mass is $1.3 \mathrm{GeV}$. Results are shown by short bars around the left end of each plot in Fig1 (Only relative differences in the vertical coordinates are relevant for the bars.) For ground state $\mathrm{P}$ waves, we obtain the largest gap between $\mathrm{PBC}$ and $\mathrm{MBC}$. Therefore, we may expect a mass shift of about $200 \mathrm{MeV}$ (500 MeV) for a ground (first exited) state when the charmonium fully dissolve.

In Fig1, we find no evidence of mass shift with such magnitudes, for neither the ground states nor the first excited states, up to a quite high temperature of about 2.3 $T_{c}$. The results for Ps charmonia are similar. This suggests that these charmonia bound state persist up to about $2.3 T_{c}$.

\subsection{Wave functions}

To check the bound-state characteristics of the charmonium states, we study the wave functions 

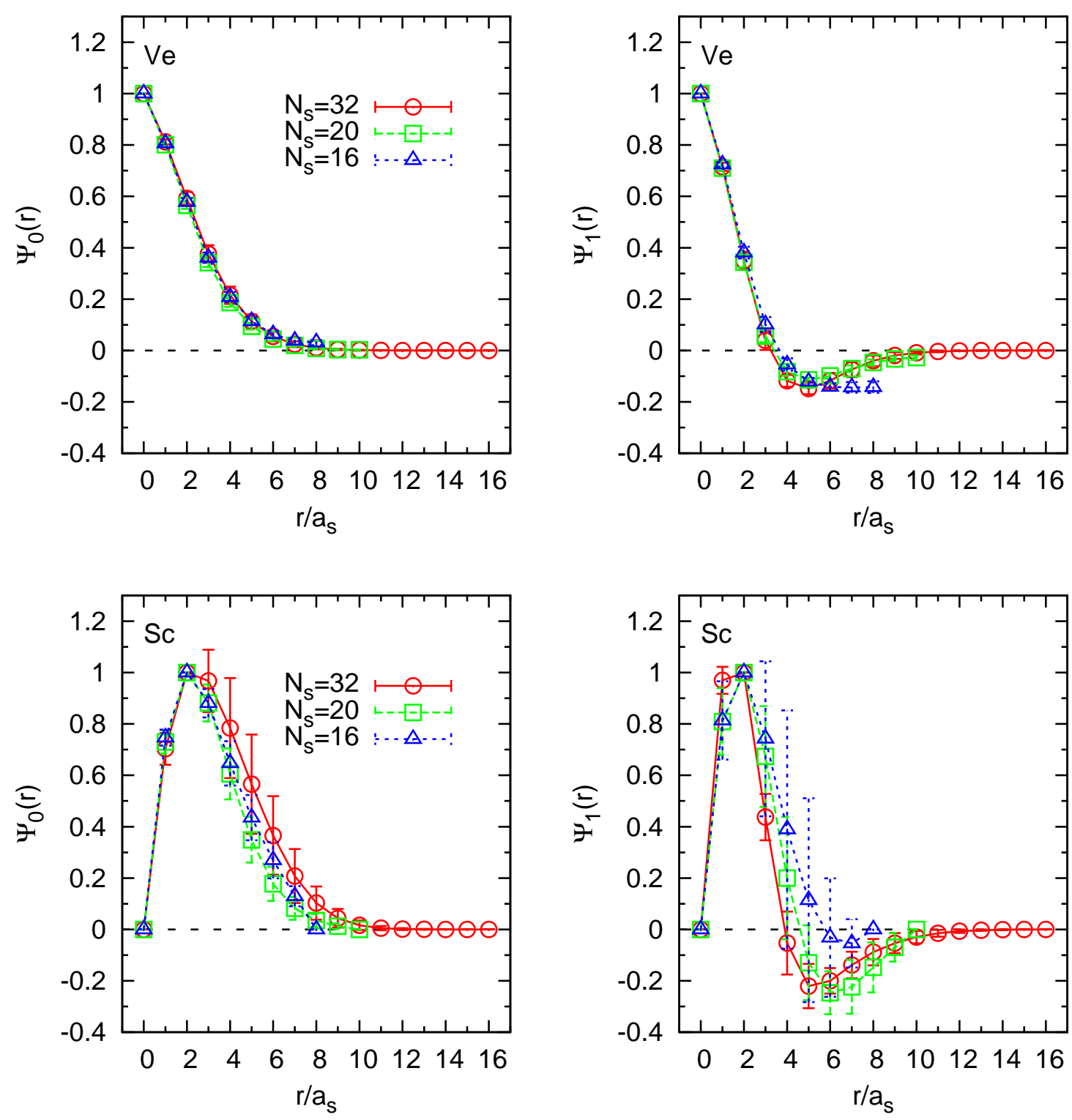

Figure 2: The wave functions for Ve (top) and Sc (bottom) charmonia at $2.3 T_{C}$. Horizontal axis is the spatial distance in the $x$-direction. Left panels show the results for ground states and right panels show those of the first excited states. Red, green and blue symbols indicate data obtained on $N_{s}=32,20$ and 16 lattices. 
corresponding to the mass eigenstates diagonalized by the multi-state variational analysis discussed in the previous subsection. When a charmonium dissociates, corresponding wave function should extend to large distances and show a sensitive dependence on the spatial size of the box. Figure 2 shows the ground state's and the first excited state's wave functions defined by (2.8) for Ve and Sc charmonia at $2.3 T_{c}$, obtained on three different spatial lattices of $32^{3}, 20^{3}$ and $16^{3}$. We find that, for all charmonia we study, the spatial size dependence is small, and the wave functions are compact. We thus find no sign of $c-\bar{c}$ scattering states up to $2.3 T_{c}$, in accordance with the observation discussed in the previous subsection.

\section{Conclusions}

We investigated if charmonia dissociates in high temperature QCD on quenched anisotropic lattices. Adopting the multi-state variational analysis to extract ground and first excited states, we examined spatial boundary condition dependence of effective masses and investigated the shape and spatial size dependence of wave functions for these states. From these studies, we found no sign of scattering states nor clear evidence of dissociation, both for the ground and first exited states of charmonia in Ps, Ve and Sc channels up to a quite high temperature of $2.3 T_{c}$, so far.

We thank the members of the WHOT-QCD Collaboration for innumerable discussions and suggestions. This work is in part supported by Grants-in-Aid of the Japanese Ministry of Education, Culture, Sports, Science and Technology (Nos. 17340066 and 19549001). Numerical calculations were performed on supercomputers at RCNP, Osaka University.

\section{References}

[1] T. Matsui and H. Satz, Phys. Lett. B 178, 416 (1986)

[2] S. Digal, P. Petreczky and H. Satz, (2001) [hep-ph/0110406]; F. Karsch, D. Kharzeev and H. Satz, Phys. Lett. B 63775 (2006) [hep-ph/0512239]

[3] T. Umeda, R. Katayama, O. Miyamura and H. Matsufuru, Int. J. Mod. Phys. A 162215 (2001) [hep-lat/0011085]

[4] T. Umeda, K. Nomura and H. Matsufuru, Eur. Phys. J. C 39S1 9 (2005) [hep-lat/0211003]

[5] M. Asakawa and T. Hatsuda, Phys. Rev. lett. 92, 012001 (2004) [hep-lat/030803]

[6] S. Datta, F. Karsch, P. Petreczky and I. Wetzorke, Phys. Rev. D 69, 094507 (2004) [hep-lat/031203]

[7] A. Jackovac, P. Petreczky, K. Petrov and A. Velytsky, Phys. Rev. D 75, 014506 (2007) [hep-lat/0611017]

[8] G. Aarts, C. Allton, M. B. Oktay, M. Peardon and J. I. Skullerud, Phys. Rev. D 76, 094513 (2007) [hep-lat/0705.2198]

[9] T. Umeda, Phys. Rev. D 75, 094502 (2007) [hep-lat/0701005]

[10] M. Lüscher and U. Wolff, Nucl. Phys. B 339, 222 (1990)

[11] H. Iida, T. Doi, N. Ishii, H. Suganuma and K. Tsumura, Phys. Rev. D 74, 074502 (2006) [hep-lat/0602008]

[12] H. Matsufuru, T. Onogi and T. Umeda, Phys. Rev. D 64, 114503 (2001) [hep-lat/0107001] 